\title{
Metamaterial-based Design for a Half Wavelength Plate in the Terahertz Range
}

Malureanu, Radu; Sun, Wujiong; Zalkovskij, Maksim; He, Qiong; Zhou, Lei; Jepsen, Peter Uhd; Lavrinenko, Andrei

Published in:

Applied Physics A: Materials Science \& Processing

Link to article, DOI:

10.1007/s00339-015-9078-3

Publication date:

2015

Document Version

Peer reviewed version

Link back to DTU Orbit

Citation $(A P A)$ :

Malureanu, R., Sun, W., Zalkovskij, M., He, Q., Zhou, L., Jepsen, P. U., \& Lavrinenko, A. (2015). Metamaterialbased Design for a Half Wavelength Plate in the Terahertz Range. Applied Physics A: Materials Science \& Processing, 119(2), 467-473. https://doi.org/10.1007/s00339-015-9078-3

\section{General rights}

Copyright and moral rights for the publications made accessible in the public portal are retained by the authors and/or other copyright owners and it is a condition of accessing publications that users recognise and abide by the legal requirements associated with these rights.

- Users may download and print one copy of any publication from the public portal for the purpose of private study or research.

- You may not further distribute the material or use it for any profit-making activity or commercial gain

- You may freely distribute the URL identifying the publication in the public portal 


\title{
Metamaterial-based Design for a Half Wavelength Plate in the Terahertz Range
}

\author{
Radu Malureanu ${ }^{1}$, Wujiong Sun ${ }^{2}$, Maksim Zalkovskij ${ }^{1}$, Qiong $\mathrm{He}^{2}$, Lei Zhou ${ }^{2}$, Peter Uhd Jepsen ${ }^{1}$, Andrei \\ Lavrinenko ${ }^{1}$ \\ ${ }^{1}$ Department of Photonics Engineering, Technical University of Denmark, Kgs. Lyngby 2800, Denmark \\ ${ }^{2}$ State Key Laboratory of Surface Physics, Key Laboratory of Micro and Nano Photonic Structures (Ministry of \\ Education), Fudan University, Shanghai 200433, China
}

\begin{abstract}
In this work a new design aimed to perform as a half-wavelength plate in the terahertz regime is presented. The fabricated samples exhibit a phase difference of 180 degrees at $0.73 \mathrm{THz}$ between the two principal polarizations that matches with the modelling results. The experimentally determined transmittances of the two polarisations were around $61 \%$, which is below theoretical predictions of reaching more than $90 \%$. The difference between the two results is explained and possibilities for increasing the transmittance are presented.
\end{abstract}

\section{Introduction}

Fundamental and application driven research in the $\mathrm{THz}$ domain with frequencies loosely defined as between 0.2 and $10 \mathrm{THz}$, is currently in rapid development [1]. Devices functioning in the $\mathrm{THz}$ range can be directly implemented in every-day applications due to favorable properties of THz radiation, including its non-ionizing nature which enables in-vivo measurements without harming for instance the exposed tissue [2]. In the same time, a multitude of molecules in the solid state vibrate in this frequency range thus enabling their detection by spectral analysis [3]. Due to these properties, devices based on $\mathrm{THz}$ radiation are approaching commercial developments, with sub-THz technology as tehtechnological basis behind e.g. full-body scanners in airports [4].

Although the possibilities of using $\mathrm{THz}$ radiation are multiple, one limitation of their widespread implementation is the difficulty in controlling the $\mathrm{THz}$ beam characteristics like bandwidth, intensity, polarization state, etc. Advances have been made in controlling the bandwidth of a THz pulse as well as its intensity and stability [4,5]. But, up to date, the polarization state of the beam is not fully controllable.

A device that helps in achieving a full control over the polarisation state is a half-wavelength plate. According to the classical definition in optics[6], the half-wavelength plate is a device, which shifts the 
relative phase between the two orthogonal linear (eigen) polarisations by $180^{\circ}[6]$. By controlling the intensity of the two orthogonal projections of the incident beam on the eigen polarizations, one can decide the azimuth of polarization of the exit beam obtained as a result of superposition of the two linearly polarized eigenwaves at the output. This performance is demonstrated by the device proposed in this article.

A numerical description of the functioning of the half-wavelength plate is as follows:

Consider an incoming incident wave having the $E$ field along the $\hat{p}$ direction as : $E=E_{0} \hat{p} e^{i(k z-\omega t)}$. We can decompose it along the two axis of the device $\left(\hat{a}_{1}, \hat{a}_{2}\right)$ :

$E=E_{0}\left(\cos \theta \hat{a}_{1}+\sin \theta \hat{a}_{2}\right) e^{i(k z-\omega t)}$, where $\theta$ is the angle between $\hat{p}$ and $\hat{a}_{1}$.

After propagating through the device, the phase difference between $\hat{a}_{1}$ and $\hat{a}_{2}$ is $180^{\circ}$ so the exit beam can be described as:

$E=E_{f}\left(\cos \theta \hat{a}_{1}-\sin \theta \hat{a}_{2}\right) e^{i(k z-\omega t)}=E_{f}\left(\cos (-\theta) \hat{a}_{1}+\sin (-\theta) \hat{a}_{2}\right) e^{i(k z-\omega t)}$

Thus, a $180^{\circ}$ phase shift induced by the device is identical to a mirroring of the incident polarisation along one of the axes of the device. We would like to note that the reciprocal statement is not valid. One can manufacture a device that rotates the incoming linearly polarised light but that is not a half-wavelength plate $[7,8]$.

Although there are already commercially available half- and quarter-wavelength plates in the THz range, they are not cost-effective and thus, further research into such devices is ongoing. An achromatic (broadband) quarter-wavelength plate have already been demonstrated [9], [10], [11]. In these cases the total thickness of the device is at least $3 \mathrm{~cm}$ thus making it potentially unfeasible for applications where the space limitations are stringent. Since the material of choice for this type of devices is quartz, the transmittivity is relatively low. Another possibility is to use a Fresnel rhomb [12]. Also in this case the transmittivity is low, but that could be overcome by using different materials. Still, the major disadvantage is the change in the optical path thus introducing further complications of the setup. THz devices that rotate the polarization of the beam have been also proposed very recently $[7,8]$. The devices reported in 
these papers have higher transmission and larger bandwidth than the one presented here but they possess a serious drawback: their functioning is limited to a specific incoming polarisation only. The output beam from the device will always have the same polarisation azimuth, independent of the incident polarisation is. Furthermore, in the case of a non-aligned incoming polarisation, the transmission of the devices will drop significantly. Thus, they cannot be referred to as half-wavelength plates.

The design presented here alleviates these potential shortcomings and functions as a true half-wavelength plate. That is, the transmitted polarisation is determined by the alignment between the incoming polarisation and the device axis, and, importantly, the output intensity is independent of the incoming polarisation If this is not the case, the device is unable to completely mirror the incoming linear polarisation, and the output polarisation will have a different azimuthal angle. This can be better understood by closely inspecting equation (1). If the two intensities are not the same at the exit, then the cosine (or sine) function will be multiplied with a term leading to an effective different rotation of the polarisation plane. To facilitate transmittance and reduce the concerns related with the material properties of the substrate we target a free-standing membrane-based structure. Theoretically, we can reach a transmittance of more than $90 \%$. Such extended behaviour comes with the price of a reduced bandwidth, as expected for any half-wavelength plate, no matter its operational range. Similar functionality based on a design of multiple stacked plates was recently published [11].

To achieve the desired functionality, we employed a metamaterials-based approach. The metamaterials field emerged more than 20 years ago [13] and has its appeal in the possibility of allowing almost arbitrary control of the optical properties of a structure at, in principle, any given wavelength [14]. We use this ability of controlling the wave behaviour inside a metamaterial to design our structure (Section 2). In Section 3 and 4 we report on fabrication and characterization of the structure. Section 5 contains discussion about the device performance.

\section{Theory and modelling}



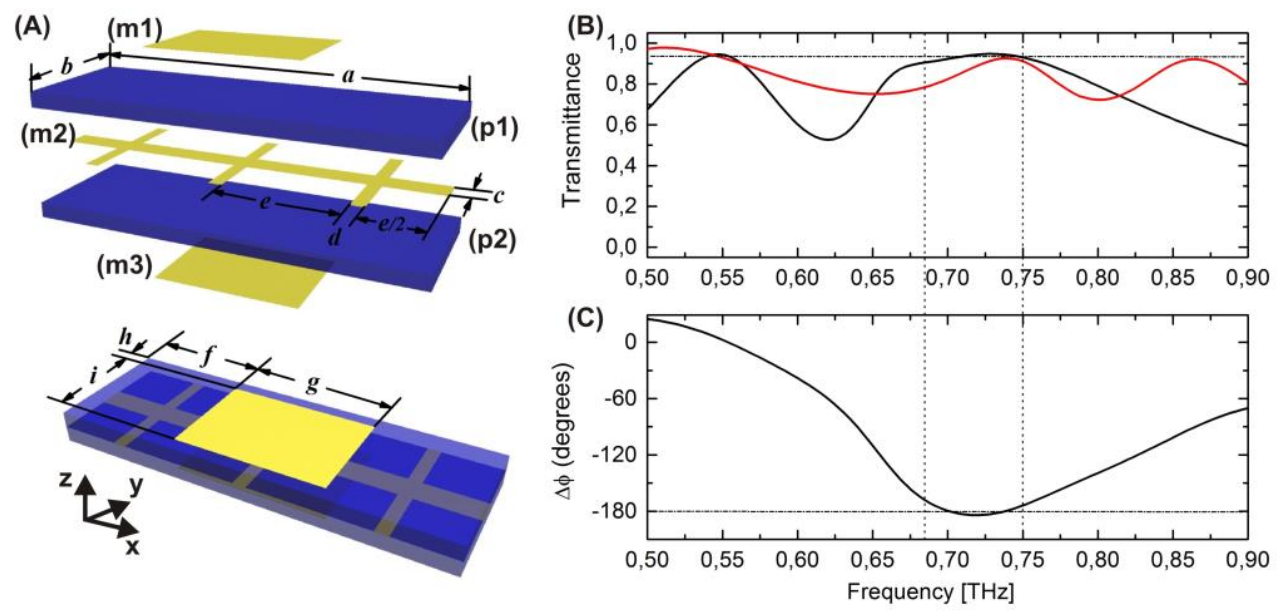

Figure 1. (A) The design of the THz sample: view of the unit cell and definition of unit cell dimensions. The optimized dimensions are given in the text; $(B)$ the transmittance of the $E_{x}$ (black) and $E_{y}(r e d)$ incident polarisations; $(C)$ calculated phase difference between the two polarizations.

To obtain a high-efficiency polarization-control device, the key task is to develop a system, where the transmittance of both eigenstates is equally high, but the mechanism to achieve it is different. In our case we control the transmission properties of the $E_{x}$ polarisation through the periodicity of the structural lattice, while the ones of the $E_{y}$ polarisation are controlled by the properties of a single unit cell through local resonances. The presented design is a result of numerical optimisation of the lattice constant and the unit cell parameters such that their individual resonances are located at the same frequency, but for two orthogonal polarisations.

We decided for a relatively simple design, where the metallic structures are rectangular patches or a simple mesh, enabling the optimization of the structure in a fast and reliable way. Also, the whole structure is symmetric, thus the computational time can be reduced. This design is not unique and other designs may be found based on the same general principle of two resonances at the same frequency obtained through different mechanisms for orthogonal polarisations. One specific advantage of this design is the absence of cross-talk. Due to its mirror-symmetry both in the $x z$ and $y z$ plane, there is no possible conversion of one polarisation to another, and thus the cross-talk is theoretically zero.

In Fig 1(a) we show the geometry of the designed structure, which consists of a metallic mesh layer $(\mathrm{m} 2)$ sandwiched by two metallic patch arrays $(m 1)$ and $(m 3)$, with two dielectric spacers $(p 1)$ and $(p 2)$ 
separating the metallic layers from direct contact. We choose this tri-layer platform in designing our $\mathrm{THz}$ waveplate because it can support multiple perfect transmission windows (in the lossless case) as long as the upper/bottom layers exhibit certain resonances governed by different principles $[15,16]$. Some dimensions are related to each other. For example, the length of a unit cell of size $a \times b$, see Fig. 1(a), is directly related to the size of the metallic patch $a=g+2 f$. Fig. 1(a) clearly indicates the anisotropy of the

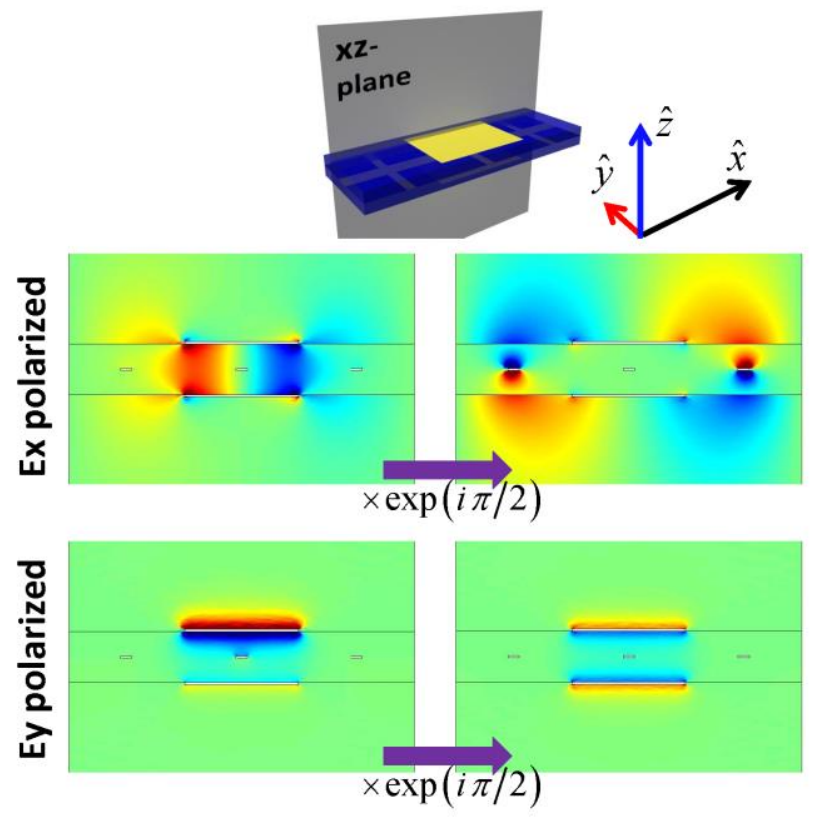

Figure 2. Distributions of $E_{z}$ components at $0.73 \mathrm{THz}$, represented in the xz-plane. (a) and (c) display the field at one same moment for $E_{x}$ and $E_{y}$ input respectively, then (b) and (d) are the situations that after quarter harmonic period of (a) and (c). The beam travels along the $z$ axis. The yz-plane wave behaviour is similar.

sample. We optimised the design for a working frequency in the $0.72-0.75 \mathrm{THz}$ range, thus the parameters of the structure are $a=300 \mu \mathrm{m}, b=100 \mu \mathrm{m}, c=22 \mu \mathrm{m}, d=10 \mu \mathrm{m}, e=90 \mu \mathrm{m}, f=$ $101 \mu \mathrm{m}, g=98 \mu \mathrm{m}, h=10 \mu \mathrm{m}, i=80 \mu \mathrm{m}$. The dielectric spacer is made from polyimide with the theoretical dielectric permittivity $\varepsilon=3.5+0.02 i$ [17] at this frequency range, and has the thickness $22 \mu \mathrm{m}$. Due to the low skin depth of aluminium at these frequencies, all metal layers are $200 \mathrm{~nm}$ thick, thus making the whole structure much thinner than the wavelength of interest. The ratio between the dimension of the structure in the $z$ direction and the free-space wavelength is approximately $1 / 9$. 
The thickness of the polyimide layers plays a critical role in the performance of the structure. In the lossless case, by carefully choosing the metal dimensions we can always obtain close to $100 \%$ transmission $[15,18]$ independent of the thickness of the structure. In real structures we have to compromise between the field enhancement that takes place near the metallic layer and the losses of the polyimide. The increase in the field enhancement also leads to increased losses thus an optimum thickness must be found.

Due to the geometry of the unit cell, it is obvious that the structure is anisotropic. We used Comsol Multiphysics to investigate the mechanisms that induce $180^{\circ}$ phase difference between two orthogonal polarizations at the working frequency. In the case of the $E_{x}$ polarisation, the field distribution at a certain moment in time is shown in Fig. 2(a). After a quarter harmonic, in Fig. 2(b), the field propagates along the $x$ direction thus the maximum intensity is now in the area of the unit cell with less metal. This type of behaviour is similar to that of a spoof surface plasmon polariton (SPP). The resonance frequency of such plasmons can be determined by the geometry of the unit cell in the propagation direction, thus by $a, g, d$, and $e$ (see Fig 1 for a description of the parameters).For $E_{y}$ polarization, the $E_{z}$ component is just oscillating in the space between the top and bottom metallic patches as shown in Fig. 2(c) and 2(d). Such behaviour leads to the conclusion that the transmission of the $E_{y}$ polarisation originates from a local resonance and depends on the values of $i$ and $b$

For a linearly polarized incident wave, we calculated the transmittance spectra $\left|S_{21}\right|^{2}$ and phase change $\varphi=\arg \left(S_{21}\right)$ in the $0.5-0.9 \mathrm{THz}$ range (see Fig. 1(b)). A transmittance exceeding $90 \%$ for both eigen polarizations is observed at $\sim 0.73 \mathrm{THz}$. In the same frequency range, the phase difference between the two eigenwaves is $180^{\circ}$ (Fig. 1 (c)), thus ensuring the functionality of the whole structure as a classical halfwavelength plate.

\section{Fabrication and measurements}

In order to fabricate the device, we established a new fabrication process-flow. For fabrication ease, we decided to use aluminium ( $\mathrm{Al}$ ) as the metal to be patterned. As opposed to our previous $\mathrm{THz}$ membranes, where the device was made entirely of nickel [19] thus would not be corroded when immersed in $\mathrm{KOH}$ 
baths to etch the Si substrate, in this case both the Al and the dielectric, polyimide, will be attacked by $\mathrm{KOH}$ [20]. To prevent $\mathrm{KOH}$ reaching the device, we first deposited a thin layer of $\mathrm{Si}_{3} \mathrm{~N}_{4}$ on the top of the wafer. This layer will protect the rest of the device in the etching stage, and then it will be dry etched.

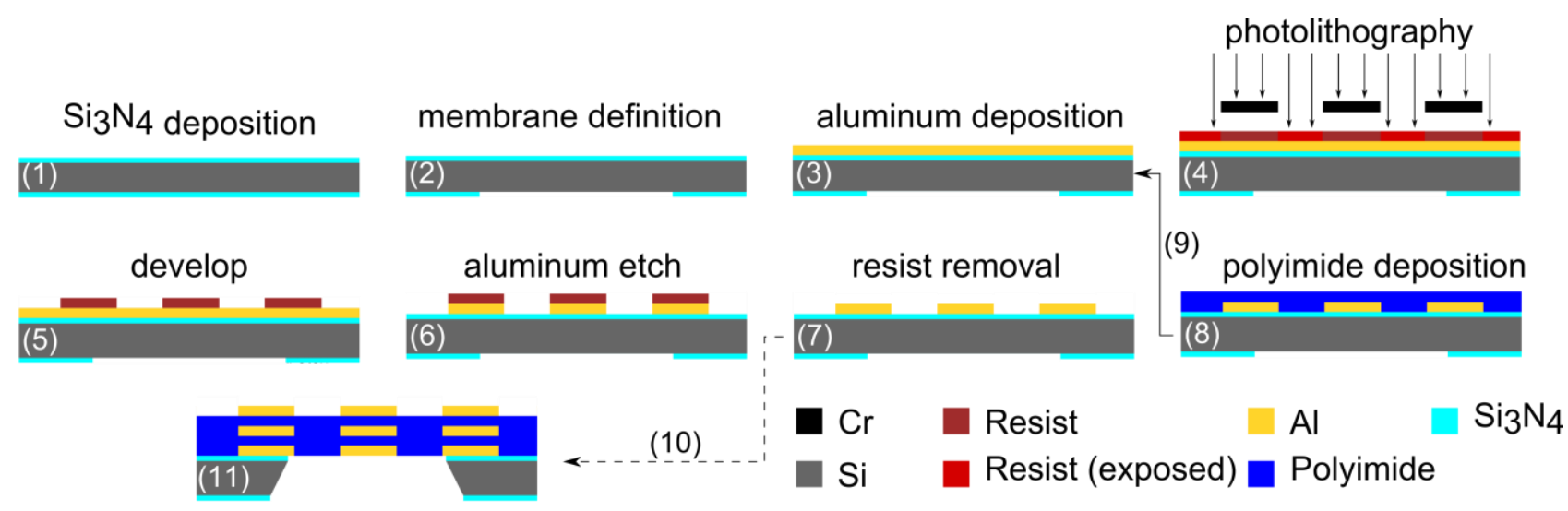

Figure 3 Schematics of the process flow. The fabrication steps are explained in the text. Not to scale

The fabrication steps are schematically shown in Fig. 3. First, we deposit a 300nm thick layer $\mathrm{Si}_{3} \mathrm{~N}_{4}$ on both sides of the Si wafer (1). We then expose, develop and dry etch the back $\mathrm{Si}_{3} \mathrm{~N}_{4}$, thus defining the position of the membranes and their dimensions (2). From this point on, all the subsequent exposures are aligned to the membrane opening.

We evaporate a $200 \mathrm{~nm}$ thick layer of $\mathrm{Al}$ on the top $\mathrm{Si}_{3} \mathrm{~N}_{4}$ (3) and, through aligned UV lithography (4), development of the resist (5) and dry plasma etching (6), we pattern the first metallic layer. We then remove the excess resist using oxygen plasma (7) and spin and hard bake the $22 \mu \mathrm{m}$ thick polyimide layer on top of the initial layer (8). Before depositing the polyimide, we optimised the spinning to obtain a thickness as close as possible to the target thickness, Thus, our spinning recipe gives an error of less than $1 \%$. Since the wavelength of interest is of hundreds of microns, the resulting $0.2 \mu \mathrm{m}$ (so less than $1 / 1000$ wavelengths) error in the fabrication has no any significant influence. In order for the polyimide layer to adhere to the substrate, a thin adhesion layer needs to be spun on the structure and the quality of this layer can greatly influence the polyimide characteristics. The process is then repeated for the second and the third metallic layers (9). After deposition of the third layer of patterned metal, we wet etch the Si wafer from the back side and dry etch the top silicon nitride layer (10), thus obtaining the final device (11). 
In Fig. 4 we show optical images of the device at various fabrication levels: (A) after the first metallic layer is patterned, step (7); (B) after processing the second layer, when it is still possible to see the first one, (C) at
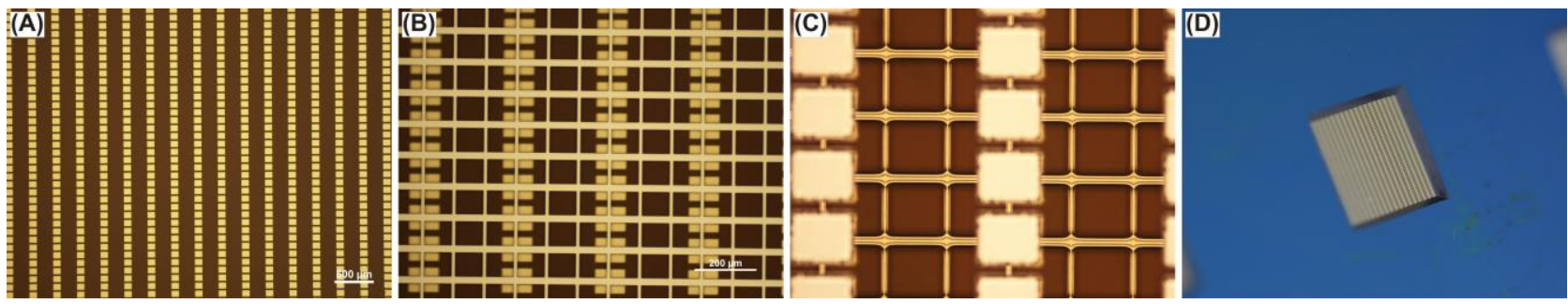

Figure 4 Optical images of the fabricated structure. (A) Overview after the first metal layer is patterned; (B) 10X magnified image of the device after the second layer patterning; (C) 20X magnified image of the final device with the focus on the second layer. The first and the third can be seen in the background and foreground, respectively. A slight misalignment between the first and the third layer can be observed; (D) Back view of the membrane. The 500 microns wafer (blue) is fully etched through.

the end of the metal patterning process. It can be seen that the alignment error of the structures is below 1 micron. Simulations (not shown here) indicate that a misalignment error below 4 microns does not influence the performance of the structure significantly. The second layer, when seen at the end of the process flow (Fig. $4($ C)), seems to have a different geometry with respect to the one seen in Fig. 4(B). This is an optical effect due to the not perfectly flat polyimide layers.

The wet etch step needs extra care. The sample is attached to a holder such that the top surface is isolated from the $\mathrm{KOH}$ bath and then immersed in the hot $\mathrm{KOH}$ solution. The temperature difference is important since it will generate stress in the structure. We kept it at the minimum by heating the bath only up to 60 degrees. Below this temperature the Si etching rate will become insignificant, thus not practical anymore, while above this temperature the stress in the structure will lead to breaking of the membrane. The reduced etching rate means that, for a standard $500 \mu \mathrm{m}$ thick wafer, it takes 22 hours to etch through. Even with all these precautions the structure is not stable, so the end of the etching process is critical in order for the membranes not to break. After the silicon is thinned to several hundreds of nanometres, the heating is stopped, thus the last part of the process is performed at decreasing temperature, helping in releasing the stress. After the etching is complete, and the membranes are free from the substrate, they are thermalized to room temperature. The final membrane dimensions are $1 \times 1 \mathrm{~cm}$ for accommodating a large $\mathrm{THz}$ spot size (Fig 4(D)). It should be mentioned that, although the process is long, lasting up to 24 hours in total, it has 
the advantage of parallelisation thus, on a 6 inch wafer one can fabricate up to 65 structures and thereby reducing the costs and increasing the yield.

\section{Characterization}

(a)

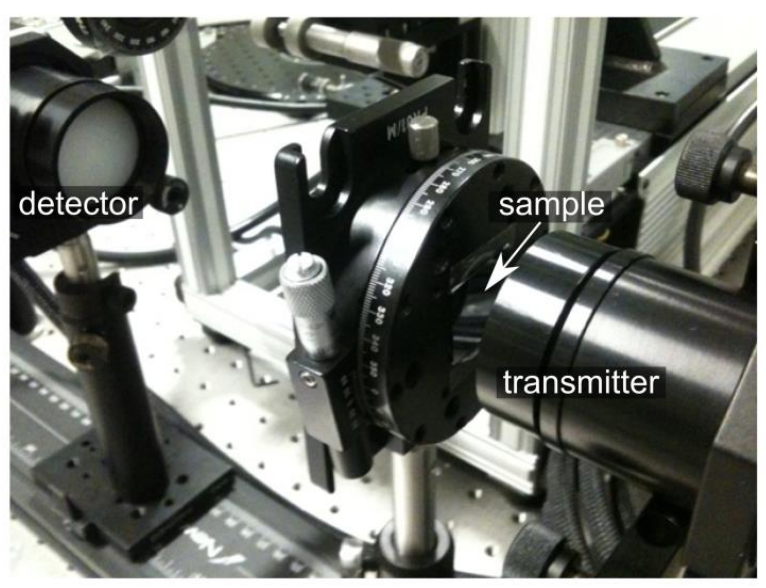

(b)

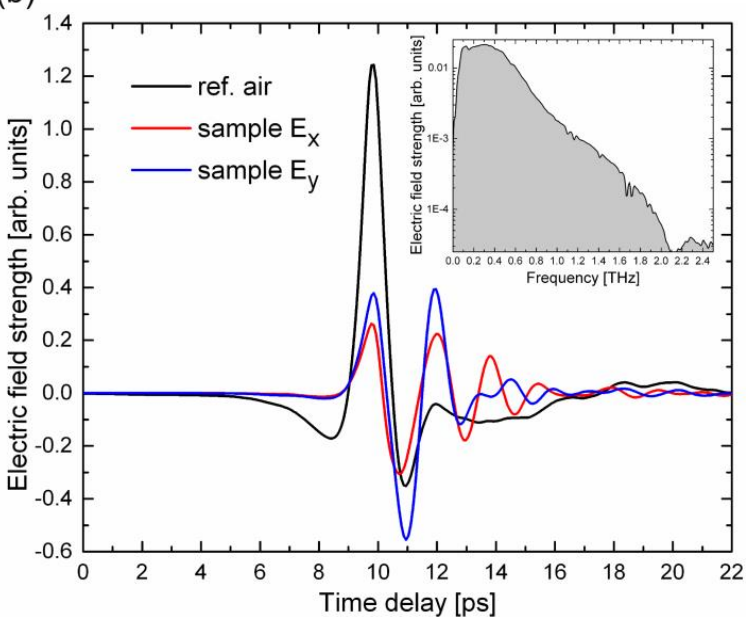

Figure 5 (A) Measurement setup. (B) THz pulse in time domain transmitted though air and half-wavelength plate. (inset)Spectrum of the THz pulse transmitted through air.

The half-wavelength plate samples were characterized using a commercial THz time-domain spectroscopy system (Picometrix T-Ray 4000), where THz pulses are both generated and detected by photoconductive switches. Every measurement is the average of 10.000 waveforms, and they were recorded at a scan rate of $100 \mathrm{~Hz}$ (total acquisition time 100s).

Fig. 5(A) shows the measurement setup. The sample is placed in the focus spot and is attached to a rotation stage. The transmission spectra were recorded for both $x$ and $y$ orientation of the half-wavelength plate. Transmission through air was used as a reference for all measurements. Fig. 5(B) displays THz pulses in the time domain transmitted through air and the half-wavelength plate. The THz pulse transmitted through air has a spectral range from $0.1 \mathrm{THz}$ to $2.0 \mathrm{THz}$, while at the frequency of interest $\sim 0.7 \mathrm{THz}$, it has a signal to noise ratio of 180 ( $45 \mathrm{~dB}$ power dynamic range). We obtain the phase and amplitude spectra using Fourier transformation of the pulses measured in the time domain [21]. Since our half-wavelength plate samples contain no supporting material, the measurements are not affected by Fabry-Perrot resonances from a substrate, and, therefore, are able to use long time-domain (100 ps) traces, which improve the spectral 
resolution of our characterization. For consistency reasons, we measured three different devices fabricated in the same batch. The results, presented in Fig. 6(A), show good reproducibility over the process flow variations. All the characterized devices have the same behaviour, and the measured phase difference at the frequency of interest is 180 degrees, as predicted by simulations (Fig. 6(B)).
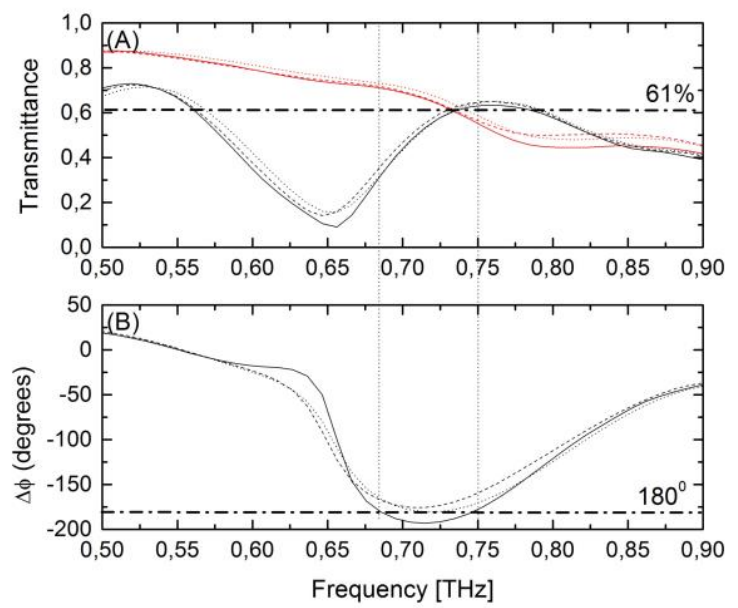

Figure 6(A) Measured transmission amplitude for the $E_{x}$ (black) and $E_{y}$ (red) polarizations. (B) Induced phase difference of the devices. The various line styles represent different samples

\section{Discussion}

The main difference between the simulated and experimental results lies in the transmission amplitude. In the simulations, the transmittance is above $90 \%$ (see Fig. 1(B)), while in experiments (Fig.6(A)) it is near $61 \%$.

We believe this difference has two origins. Firstly, the simulations assume perfectly flat layers and neglect the surface roughness inherent in any fabrication process. Scattering definitely lowers transmission values. However, the roughness values are much below the functioning wavelength (approximately $0.4 \mathrm{~mm}$ ), and we generally expect that it will have measurable but marginal influence on the signal. This influence cannot explain a $30 \%$ decrease in transmission. We estimate that the main factor in this drop comes from an underestimated absorption of the polyimide layer. The permittivity value we used in the simulation $\varepsilon=3.5+0.02 i$ was taken from literature [17]. We performed additional simulations to account for possible higher losses of the polyimide layers. The results illustrated in Fig. 7, show that the increase in 
losses does not change the working frequency and phase difference, but rather diminishes the transmitted signal.
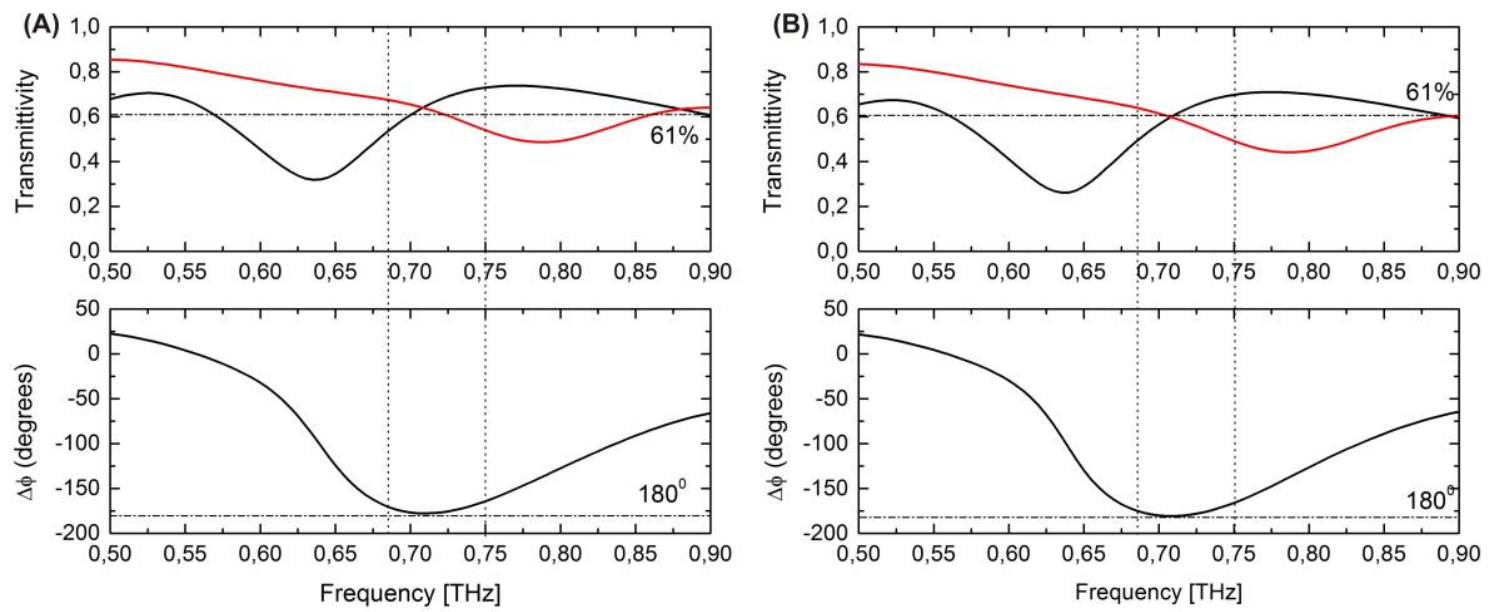

Figure 7 Simulated transmission values and phase differences when the permittivity of polyimide is $3.5+0.3 i(A)$ and $3.5+0.35 i(B)$. The dashed lines represent the frequency interval and the values obtained in experiments.

According to these results, in order to explain the lower level of transmitted signal, we need to adopt the imaginary part of the relative permittivity of polyimide between 0.3 and 0.35 , which amounts to a pronounced 15 times increase from the 0.02 value initially stated. At $0.7 \mathrm{THz}$, an imaginary permittivity of 0.3 corresponds to an absorption coefficient of $20 \mathrm{~cm}^{-1}$, in very good agreements with broadband measurements by Cunningham et al.[22]. Differences may also arise due to the fabrication process, especially in the baking stage that may alter the dielectric properties of polyimide. Additionally, we used an adhesion layer to ensure the stability of the polyimide deposition [23]. This layer may introduce additional losses not taken into account in the model. Apart from these effects, other factors that influence the transmittance are non-uniformity of the polyimide layer and the roughness of the metallic structures. It is worth to mention that increases in losses of one order of magnitude for this type of polyimide are also mentioned in literature $[17,24]$. As a possible solution, polyimide can be substituted by benzocyclobutene commonly known as $B C B$. BCB is a polymer that can be spun in thick layers and has low experimental losses in the THz range $[25,26]$. Another substitute for polyimide can be TOPAS that has extremely low loss in the THz range[27] 


\section{Conclusion}

In this work we have designed, simulated, fabricated and characterized a metamaterial-based membrane that acts as a half-wavelength plate in the $\mathrm{THz}$ regime. Its performance corresponds to the definition of the classical half-wavelength plate in optics, and it can be used for rotating an incoming linearly polarised $\mathrm{THz}$ beam with an arbitrary angle. We employ two different mechanisms of eigenwave propagation to equalize their transmittance and simultaneously impose a 180 degrees phase difference between the eigenwaves in the desired frequency range. Characterization successfully confirms designed parameters. The waves experience a half-wavelength relative phase shift and meanwhile have the same transmission amplitude. The difference in transmittance between the theoretically predicted value of above $90 \%$ and experimental one of $61 \%$ is most probably due to higher than typically assumed losses in the dielectric layers. Modelling of systems with higher polyimide losses confirms this assumption. By utilizing dielectrics such as $B C B$ and TOPAS with lower losses we can significantly improve the transmission characteristics.

\section{Acknowledgments}

RM, MZ, PUJ and AL acknowledge partial financial support from the FTP THzCOW project. WJS, QH and LZ thank financial supports from the National Science Foundation of China (11174055, 11204040), the Program of Shanghai Subject Chief Scientist (12XD1400700) and the China Postdoctoral Science Foundation (2012M520039, 2013T60412). 


\section{References}

1. P. U. Jepsen, D. G. Cooke, and M. Koch, Laser Photon. Rev. 5, 124 (2011).

2. E. Pickwell, B. E. Cole, A. J. Fitzgerald, M. Pepper, and V. P. Wallace, Phys. Med. Biol. 49, 1595 (2004).

3. B. Ferguson and X.-C. Zhang, Nat. Mater. 1, 26 (2002).

4. M. Tonouchi, Nat. Photonics 1, 97 (2007).

5. M. C. Hoffmann and J. A. Fülöp, J. Phys. D. Appl. Phys. 44, 083001 (2011).

6. A. Yariv and P. Yeh, Optical Waves in Crystals : Propagation and Control of Laser Radiation (2003).

7. L. Cong, W. Cao, X. Zhang, Z. Tian, J. Gu, R. Singh, J. Han, and W. Zhang, Appl. Phys. Lett. 103, 171107 (2013).

8. N. K. Grady, J. E. Heyes, D. R. Chowdhury, Y. Zeng, M. T. Reiten, A. K. Azad, A. J. Taylor, D. A. R. Dalvit, and H.-T. Chen, Science 340, 1304 (2013).

9. J.-B. Masson and G. Gallot, Opt. Lett. 31, 265 (2006).

10. A. K. Kaveev, G. I. Kropotov, E. V Tsygankova, I. A. Tzibizov, S. D. Ganichev, S. N. Danilov, P. Olbrich, C. Zoth, E. G. Kaveeva, A. I. Zhdanov, A. A. Ivanov, R. Z. Deyanov, and B. Redlich, Appl. Opt. 52, B60 (2013).

11. M. Nagai, N. Mukai, Y. Minowa, M. Ashida, J. Takayanagi, and H. Ohtake, Opt. Lett. 39, 146 (2014).

12. J. Shan, J. I. Dadap, and T. F. Heinz, Opt. Express 17, 7431 (2009).

13. J. B. Pendry, A. J. Holden, D. J. Robbins, and W. J. Stewart, IEEE Trans. Microw. Theory Tech. 47, 2075 (1999).

14. B. Wood, Laser Photonics Rev. 1, 249 (2007).

15. L. Zhou, W. Wen, C. Chan, and P. Sheng, Phys. Rev. Lett. 94, 243905 (2005).

16. W. Sun, Q. He, J. Hao, and L. Zhou, Opt. Lett. 36, 927 (2011).

17. H. Tao, N. I. Landy, C. M. Bingham, X. Zhang, R. D. Averitt, and W. J. Padilla, Opt. Express 16, 7181 (2008).

18. R. Malureanu, M. Zalkovskij, Z. Song, C. Gritti, A. Andryieuski, Q. He, L. Zhou, P. U. Jepsen, and A. V Lavrinenko, Opt. Express 20, 22770 (2012). 
19. M. Zalkovskij, R. Malureanu, C. Kremers, D. N. Chigrin, A. Novitsky, S. Zhukovsky, P. T. Tang, P. U. Jepsen, and A. V. Lavrinenko, Laser Photon. Rev. 7, 810 (2013).

20. K. R. Williams, K. Gupta, and M. Wasilik, J. Microelectromechanical Syst. 12, 761 (2003).

21. R. Malureanu, A. Lavrinenko, D. G. Cooke, P. U. Jepsen, S. Xiao, and L. Zhou, in Conf. Lasers Electro-Optics 2010 (OSA, Washington, D.C., 2010), p. CTuF7.

22. P. D. Cunningham, N. N. Valdes, F. A. Vallejo, L. M. Hayden, B. Polishak, X.-H. Zhou, J. Luo, A. K.-Y. Jen, J. C. Williams, and R. J. Twieg, J. Appl. Phys. 109, 043505 (2011).

23. H. Microsystems, PI-5878G Wet Etch Applications (2009), p. 11.

24. H. Tao, C. Bingham, A. Strikwerda, D. Pilon, D. Shrekenhamer, N. Landy, K. Fan, X. Zhang, W. Padilla, and R. Averitt, Phys. Rev. B 78, 241103 (2008).

25. O. Paul, C. Imhof, B. Reinhard, R. Zengerle, and R. Beigang, Opt. Express 16, 6736 (2008).

26. E. Perret, N. Zerounian, S. David, and F. Aniel, Microelectron. Eng. 85, 2276 (2008).

27. H. Bao, K. Nielsen, H. K. Rasmussen, P. U. Jepsen, and O. Bang, Opt. Express 20, 29507 (2012). 